Spencley, R., Pfeffer, G., Gordon, E., Hain, F., Reed, D., and Marosszeky, M. (2018). "Behavior-Based Quality Closing the Knowing Doing Gap." In: Proc. $26^{\text {th }}$ Annual Conference of the International. Group for Lean Construction (IGLC), González, V.A. (ed.), Chennai, India, pp. 1170-1181. DOI: doi.org/10.24928/2018/0532. Available at: www.iglc.net

\title{
BEHAVIOR-BASED QUALITY, CASE STUDY OF CLOSING THE KNOWING-DOING GAP
}

\author{
Rodney Spencley ${ }^{1}$, George Pfeffer ${ }^{2}$, Elizabeth Gordon ${ }^{3}$, Fritz Hain ${ }^{4}$, \\ Dean Reed $^{5}$ and Marton Marosszeky ${ }^{6}$
}

\begin{abstract}
This is a case study of a large US general contractor's efforts to rethink and implement a new behavior-based approach to quality to achieve zero errors, zero defects, zero rework, and zero surprises. This GC has a long history of building a culture of Behavior-Based Safety and has approached quality the same way. Recognition of upstream behaviors that resulted in quality issues and unpredictable results during construction led to a focus on changing the mindset and behaviors of all project stakeholders to enable the team to achieve the intended results. While owners and designers have an indirect connection to safety results, their behavior and actions directly affect quality outcomes. Although developed independently of Quality Function Deployment (QFD), this GC's approach is similar. Its approach focuses on understanding the customer's expectations and what is required technically in detail from suppliers to achieve them. It focuses on understanding and describing in technical terms what are the 'distinguishing' features of the work from each stakeholder's perspective, and on aligning its teams on measurable acceptance criteria to achieve customer expectations. This process for making knowledge explicit in order to agree on what quality means to the customer allows the team to fabricate and install its products correctly in such a way as to close the 'knowing-doing' gap that plagues most companies and projects.
\end{abstract}

\section{KEYWORDS}

Quality, workflow, indicators, Behavior-Based Safety (BBS), Behavior-Based Quality (BBQ), Quality Function Deployment (QFD), Rework

1 Director for Quality, DPR Construction, 1450 Veterans Boulevard, Redwood City, CA 94063, USA, RodneyS@dpr.com

2 President, DPR Construction, 1450 Veterans Boulevard, Redwood City, CA 94063, USA, GeorgeP@dpr.com

3 Quality Leader, DPR Construction, 1450 Veterans Boulevard, Redwood City, CA 94063, USA, ElizabethG@dpr.com

4 RISQ Group Leader, DPR Construction, 2000 Aerial Center Parkway, Suite 118, Morrisville, NC 27560, USA, FritzH@dpr.com

5 Lean/Integration Advocate, DPR Construction, 1450 Veterans Boulevard, Redwood City, CA 94063, USA, DeanR@dpr.com

6 Managing Director, Marosszeky Associates P.L., 40 Maligan Lane, Mandalong NSW 2264, Australia, marton@marosszekyassociates.com 


\section{INTRODUCTION}

A few years ago, during a continuous improvement conversation, one of the General Contractor's (GC's) Business Unit Leaders recognized a disconnect: there were many Zero-Defect letter shanging on the walls in the office, but they were still going back to fix work. Some of those projects experienced simple warranty calls; others involved substantial rework. It appeared that something wasn't working. Rodney Spencley, the director for safety and quality, began investigating and found that actual quality results across the company didn't adequately align with the company's definition of success:

- Sometimes they delivered work right the first time to very satisfied customers.

- Sometimes they achieved Zero Defects at substantial completion but spent extra time and money in doing so.

- Sometimes there were warranty call backs.

- Sometimes they delivered work in accordance with the plans and specifications only to have the owner or architect view the work and say, "that wasn't what I was expecting, tear it out."

In response, the GC's leadership committed to doing something different. They developed an approach to quality much like their approach to safety: a behavior-based approach that wasn't hierarchical or bureaucratic, but instead inspired and motivated people to think and act differently. This paper describes the approach and its application to several case studies.

\section{THE PROBLEM}

Rework, or "the waste of correction" was first identified as a key waste by Ohno (1988).Rework has always plagued the construction industry. Research suggests that rework amounts to anywhere from $2 \%$ to $12 \%$ of project value (Burati et al. 1992; Dougherty et al. 2012, Ledbetter 1994, Love 2002, Marosszeky 2002, Thomas 2003). Marosszeky and Thomas reported on a 2002-3 study in which the direct and indirect costs of rework incurred by the general contractor and subcontractors was measured through the analysis of 3,500 defects on $\$ 60$ million of construction activity spread over four projects. The Australian Centre for Construction Innovation(ACCI) research team (Thomas, 2003) reported that total rework cost ranged between $3.4 \%$ and $6.2 \%$ of project value, of this the direct rework costs represented only some $60 \%$ while the balance was the indirect cost of organising the rework.

In 2012, the Navigant Construction Forum ${ }^{\mathrm{TM}}$ (Dougherty 2012) reported a much higher range of costs for rework:

- Survey responses estimated the direct cost for rework in the range from $4.03 \%$ to $6.05 \%$ of project value and it was estimated that these should be marked up $80 \%$ to account for associated indirect costs,

- Survey responses estimated that on average the schedule grows by $9.82 \%$ due to rework 
Rodney Spencley, George Pfeffer, Elizabeth Gordon, Fritz Hain,

Dean Reed and Marton Marosszeky

- One-third of survey respondents in a 2011 Construction Industry Institute (CII) study believed that that their recorded rework was only $50-75 \%$ of actual rework experienced

The ACCI research examined the causes of this problem in some detail, primarily speaking to general and subcontractor management and workers. The research team identified the following challenges to the achievement of quality.

- Lack of consistent standards, tools and procedures between projects

- Limited continuity/transfer of knowledge and procedures between project teams

- Fragmentation of the supply chain makes it harder to manage work but easy to shift blame

- Management attitudes and competition between teams makes it hard to introduce change

- It is difficult to train subcontract workers and to manage the quality of their work

- The costs of post-project completion rework are generally hidden by transferring the cost to the next job

- It was found that none of the subcontractors interviewed considered the following trade to be a customer

The ACCI research team concluded that in summary, the problem was not that project teams were unable to get quality right, they felt that the skills and knowledge were available, rather the problem was that project teams were hampered by the above, which impacted their ability to get it right every time.

\section{MISALIGNED BEHAVIORS}

Safety and quality reflect a similar disconnect between ambitions and results. In safety, even though nobody wants to see anyone injured, injuries still occur. In quality, even though everyone is committed to producing acceptable quality work, unacceptable work is routinely produced. In both cases, ambitions are aligned, but behaviors, knowledge, and practices are not.

This GC has a long history of applying Behavior-Based Safety in its business. (Trethewyet al. 2000). When Rodney Spencley, the company's safety lead recognized that they could install work in accordance with the plans and specifications and still have stakeholders unhappy with the end result, he realized that, much like safety, they needed to look more deeply at upstream behaviors and communications to affect change. Different parties have a critical influence on achieving safety and quality outcomes. For safety, the general contractor's leadership and behaviors directly influence the culture and safety results. Owners and architects indirectly affect safety through the requirements they set and how actively they support the general contractor's safety culture and program. In contrast, the behaviors of the owner, architect and engineers critically and directly influence the project's quality outcomes.

To Rodney, the industry's existing quality approach was too ambiguous and too focused on lagging indicators. Lagging indicators track misalignment of expectations after work has been delivered. Typical examples from compliance-based efforts (think 
"thou shalt") include failed inspections, issues documented after a first-installed review, and issues discovered during a quality walk. This approach can be best described as ' $d o$ work, check work, put issues on a list, and redo the work'.

Many industry quality programs focus on requiring a higher level of documentation to achieve zero rework, zero errors and zero defects. Many quality management systems document lessons learned and feed that knowledge upstream to project teams at regular intervals throughout a project, such as at design reviews, submittal reviews, mock-ups, and first-installations. At these milestones, teams check work received from the design professional, trade partners, and the craft against a database of knowledge. But it's often overlooked that relying on a database of lessons learned and a lot of checklists will not guarantee a quality product. This approach alone has been unsuccessful in delivering the quality that the customer expects.

\section{THE SOLUTION}

\section{LANGUAGE AND PERSPECTIVES}

Marton Marosszeky interviewed Rodney for his book, Total Construction ManagementLean Quality in Construction Project Delivery, and described his approach. "Rodney sees that a major challenge in achieving quality objectives is the absence of a common language among the parties. The owner's language is about costs and key operational and aesthetic outcomes; the architect's language is primarily focused on the aesthetic, and end-user functionality; the fire engineer's language is about flame spread; the structural engineer's language is about structural integrity, etc. There are so many different languages being spoken that inevitably everyone is having a different conversation, even though they may be in one room." Although stakeholders sometimes have common words to bridge the gap between these many different languages, stakeholders often come from different backgrounds, have different perspectives, and likely have different motivations. So these common words mean different things to each stakeholder. Without spending time to clearly define measurable acceptance criteria for each expectation, the stakeholders each have unaligned assumptions and expectations. "The result is that some of the owner's key functional concerns are lost in the babble." (Oakland and Marosszeky 2017)

\section{UNDERSTANDING EXPECTATIONS}

The starting point and primary focus of this GC's approach has been to work as closely as possible with project owners and designers to understand their requirements and expectations of the work. Initially, stakeholder expectations may be abstract, but a series of alignment conversations that focus on what quality means to the delivery team members in light of the customer's expectations move stakeholders away from the abstract and result in aligned measurable acceptance criteria for each expectation. This is a process for moving from the ambiguous unknown to the defined. Alignment activities can be monitored and tracked as leading indicators that evidence the focused effort on meeting quality expectations. (Oakland and Marosszeky 2017). Leading indicators measure the likelihood of success. For example, if the team has agreed on acceptance 
criteria before beginning work on a deliverable, then there is a higher likelihood that expectations will be met and there will be no surprises when the work is complete.

Although this approach was not inspired or informed by knowledge of the "Quality Function Deployment" (QFD) developed in 1972 at Mitsubishi's Kobe shipyard, it shares the intention to deeply understand customer requirements, these are then translated into language and objectives that are clear to all those responsible for producing the work, supported by measurable criteria that support everyone within the supply chain engaged in realizing them (Hauser and Clausing 1988).

And similar to Behavior-Based Safety, the focus of quality management is shifted to the behaviour and knowledge/understanding of people: what those representing the owner, design team, general contractor and trade contractors want, know, and believe should be done. All are stakeholders in quality.

Foundationally, it's about initiating conversations to identify what each stakeholder believes is important or "distinguishing" about a system or building element consistent with the customers' expectations. We call these "Distinguishing Features of Work" (DFOW). It's okay for every stakeholder to have different viewpoints on what's distinguishing. But once a DFOW is identified, the stakeholders must discuss and agree on what quality means for that piece of work. We call this process developing "Measurable Acceptance Criteria."

\section{A TWO-PRONGED APPROACH: PRESCRIPTIVE AND DESCRIPTIVE}

Quality requirements generally fall in two categories: prescriptive and descriptive. The construction industry has historically focused almost solely on prescriptive, or compliance-related, expectations. This typically includes testing and inspecting to verify that construction meets applicable building code and the requirements in the drawings and specifications. The owner, designers, general contractor, and trade partners tend to overlook descriptive expectations, however, which involve developing and agreeing on objective criteria to measure the aesthetic/craftsmanship elements of quality.

For example, prescriptive expectations for a curtain wall system will typically include verifying materials, periodically inspecting welds, performing water testing, and verifying consistent sealant joint widths around penetrations. Descriptive expectations could include those same sealant joints having no blemishes visible from an agreed upon distance.

While recognizing that this two-pronged approach to quality must be scalable to various sizes and types of projects, and stakeholder involvement, this GC strives to make it an intentional process that starts during the pursuit phase and is carried through preconstruction and construction.

\section{POINTS OF RELEASE}

A concept that underpins this two-pronged approach is called "Point of Release," which Digby Christian (2012) of Sutter Health identified as the point when work is released for prefabrication or purchase. Digby explained in remarks celebrating achievement of a major completion milestone for the Sutter Health Eden Medical Center 
Hospital that all design, code compliance and coordination questions must be answered to minimize lost time and increased costs due to rework.

The GC's quality leaders recognized that the Point of Release for quality was in fact many hand-offs from design through construction, commissioning and building turnover. For quality conversations to be useful, they need to happen before and account for each Point of Release. If the team has aligned on measurable acceptance criteria for the deliverable and the deliverable meets the agreed to acceptance criteria, the risk in releasing the work has been minimized. If the alignment activities did not occur, however, the hand-off becomes a point of contention, instead of a Point of Release.

\section{THE QUALITY WORKFLOW}

This GC's approach can be broken down into the four steps shown in Figure 1.

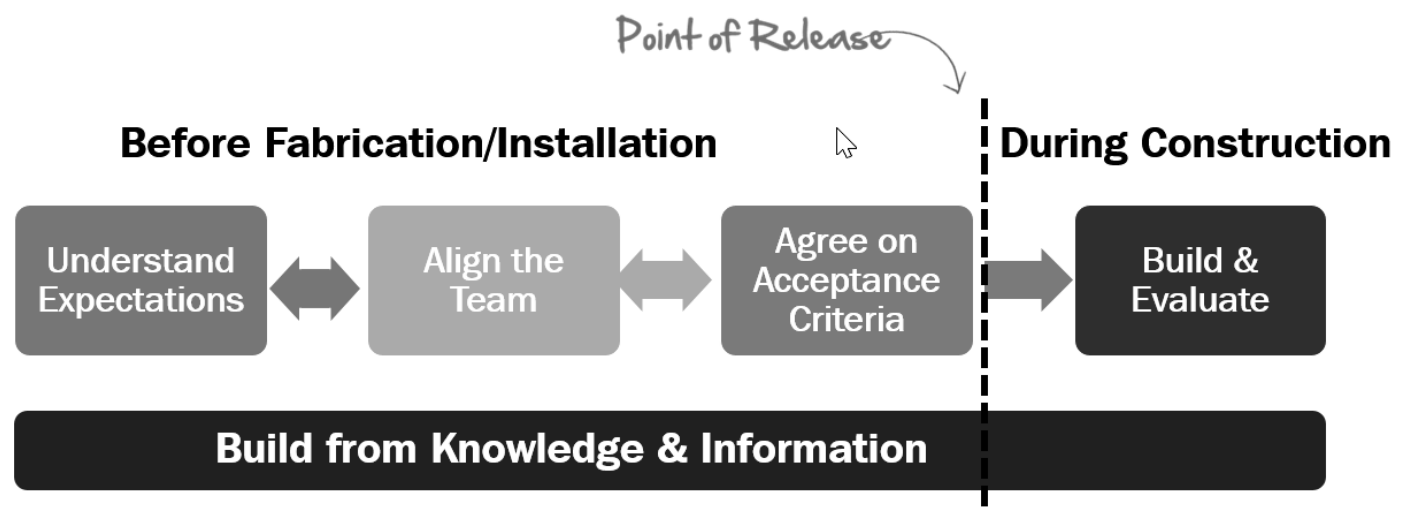

Figure 1: The four steps to quality.

- Understand expectations. Educate project stakeholders on the vision of no surprises, and develop a preliminary list of Distinguishing Features of Work (DFOW). DFOW should include common issues that usually need to be resolved in the design documents before construction; those features of the work that have caused problems in the past; common sources of rework; new or unique features of the work; and those areas that require increased attention in order to achieve the intended result. Then, after compiling an initial list of DFOW, agree on a path forward for developing measurable acceptance criteria for both the prescriptive and descriptive elements of quality.

- Align the team. Every workgroup should fall under a shared leadership group. Depending on the project, these workgroups could be set up by phase, area, or scope of work. Every team member must know who the accountable lead is for the owner, designer, builder, and any major trade partners for their particular work product, so they can understand and help achieve aligned acceptance criteria for that work. This "4 in a Box" concept (or essentially the number of major stakeholders in the DFOW supply chain) should be in place from the lowest to highest levels of the project organization. It's power flows from forcing joint accountability, which is required to achieve a high performing team. 
- Agree on measurable acceptance criteria before starting work or releasing key deliverables. The project stakeholders should understand how both descriptive and prescriptive expectations for key features of work need to be communicated in the construction documents and how they will be evaluated and signed-off during construction.

- Verify that the delivered product complies with the agreed upon acceptance criteria. As acceptance criteria become more complex, a mock-up should be built. When evaluating the mock-up to the initial acceptance criteria, evaluation of the work should fall into three categories 1) it meets the acceptance criteria; 2) the owner or design team would like to change the measurable acceptance criteria and all stakeholders agree that the change should be made; or 3) it does not meet the agreed to acceptance criteria and should be redone in which case, the team should gather together to understand the root cause,develop a strategy to mitigate the situation, and learn from the shortcoming, so as not to repeat the same mistakes.

\section{CLOSING THE KNOWING-DOING GAP}

Most companies suffer from an inability to convert what they know into action, which Stanford professors Jeffrey Pfeffer and Robert I. Sutton (1999)labelled the "KnowingDoing Gap." Hauser and Clausing (1988) in writing of the challenge in converting ideas into reality say "None of this is simple. An elegant idea ultimately decays into process, and processes will be confounding as long as human beings are involved. But that is no excuse to hold back. ... What is also not simple is developing an organization capable of absorbing elegant ideas. The principal benefit of the house of quality is quality in-house. It gets people thinking in the right directions and thinking together. For most U.S. companies, this alone amounts to a quiet revolution".

The Quality Workflow method seeks to close the Knowing-Doing Gap, or to convert what is known into action. It's a method in which the customer, design team, and construction stakeholders share their expectations and knowledge and agree on acceptance criteria for important product features, so they can be fabricated and installed correctly. Knowledge becomes explicit and this creates the basis for doing the right things at the right time.

This GC recognizes that not all project owners and designers will fully engage in this four-step approach to quality. Nonetheless, they are committed to advancing the vision of predictable results by striving to align the behaviors, knowledge, understanding and practices of all stakeholders. Experience has shown that the greater the engagement by all project stakeholders, the greater the chances that the end result will be a high-quality project.

\section{RESULTS SO FAR}

Wherever project teams have embraced the Quality Workflow early in the project-even for just a few scopes of work - they have delivered high quality work with no surprises. The outcomes from three projects have inspired other teams to shift to the new approach. 


\section{UNIVERSITY CENTER FOR CONVERGENT BIOSCIENCE, EXTERIOR GROIN VAULT STORY}

The University community had high performance expectations for this 190,000 square foot project that included labs, Class 100 and 1,000 nanotechnology clean rooms, imaging suites, and conference and classrooms. The building would "bring together research in engineering and biomedical sciences to fast-track the detection and cure of diseases." ("USC Michelson Center for Convergent Bioscience" 2017). The University also had high aesthetic expectations for the exterior façade. They needed the building to match the surrounding historical buildings and blend seamlessly with campus aesthetics. The team applied The Quality Workflow to the exterior brick groin vaulted ceilings, a DFOW of the campus and to the project architect, which resulted in zero rework and a celebrated element of the project.

Through a series of conversations, they were able to identify DFOW from each stakeholder's perspective and align on measurable acceptance criteria. For example, the random brick color was very important to the University. They discovered that each stakeholder had different expectations as to what the common term "random" brick colormeant. Their trade partner and the GC thought random meant pulling bricks from the pre-blended boxes and installing them. To the campus architect, it meant there would not be any dark spots or dark stripes. Finally, to the project architect it meant that the pattern would match the look and feel of the mock-up. Ideally, these conversations and development of acceptance criteria should have happened before constructing the mockup. Although there were different expectations, the group agreed before starting production work that the measurable acceptance criteria for random color, meant that no more than six dark colored bricks would be touching. This required the bricklayers to pay special attention when they pulled the veneer bricks from the pre-blended boxes.
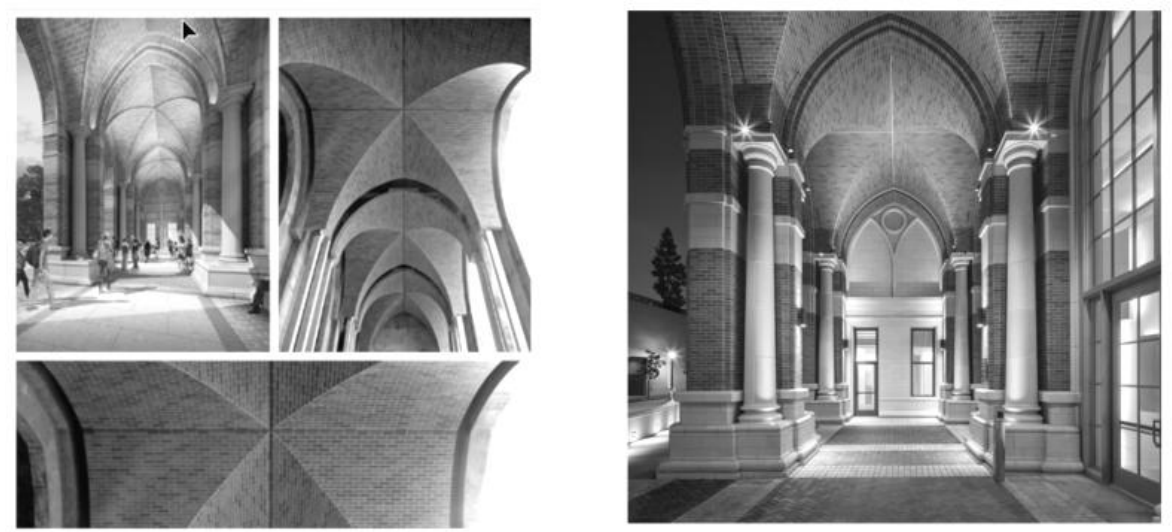

Figure 2: Center for Convergent Bioscience, exterior groin vault.

Even though this expectation was uncovered after the scope of work was procured, the GC's trade partner said that executing this expectation would not cost anymore because they could communicate these requirements to their foreman ahead of time. The foreman had the acceptance criteria and verbally communicated expectations to the craft 
Rodney Spencley, George Pfeffer, Elizabeth Gordon, Fritz Hain,

Dean Reed and Marton Marosszeky

during morning huddles. The GC found that the most effective communication of expectations is person-to-person. When the designers reviewed the installed work, there were no surprises and they were very happy with the result.

The Instagram post by the President of the University mid-way through construction appears on the left in Figure 2: "We're making excellent progress on the construction of USC Michelson Hall - the entry vaults, are now installed (Top-R and Bottom [photo]). On the Top-L, you can see what it will look like when it's completed! Fight On! \#USC \#FightOn." The completed work can be seen in the photo on the right in Figure 2.

\section{University Medical Center}

The University Medical Center project included construction of an emergency department and an eighteen-story patient tower, in two phases. The team completed Phase 1 under a more traditional approach to quality and achieved a Zero-Defect Letter. Quality activities focused on documenting, tracking, and closing out issues during construction. But, when the GC team heard how their company was shifting its quality focus to DFOW and measurable acceptance criteria, they recognized an easier and more efficient way to achieve Zero Defects.

The team launched the new quality approach on the Phase 2 interiors and realized even better results. The architect saw how valuable these conversations were and offered to bring the entire design team into the quality conversations. The stakeholders worked together to make sure everyone's expectations were reflected in the construction documents.

The results were fascinating. For the elevator lobbies, which were identified as a DFOW, only eight Requests for Information (RFIs)were needed before work started. And zero RFIs were issued after work started. Trade partners also realized increased field productivity. The team originally planned for 53 work days on the elevator lobby; the actual duration was 32 days.
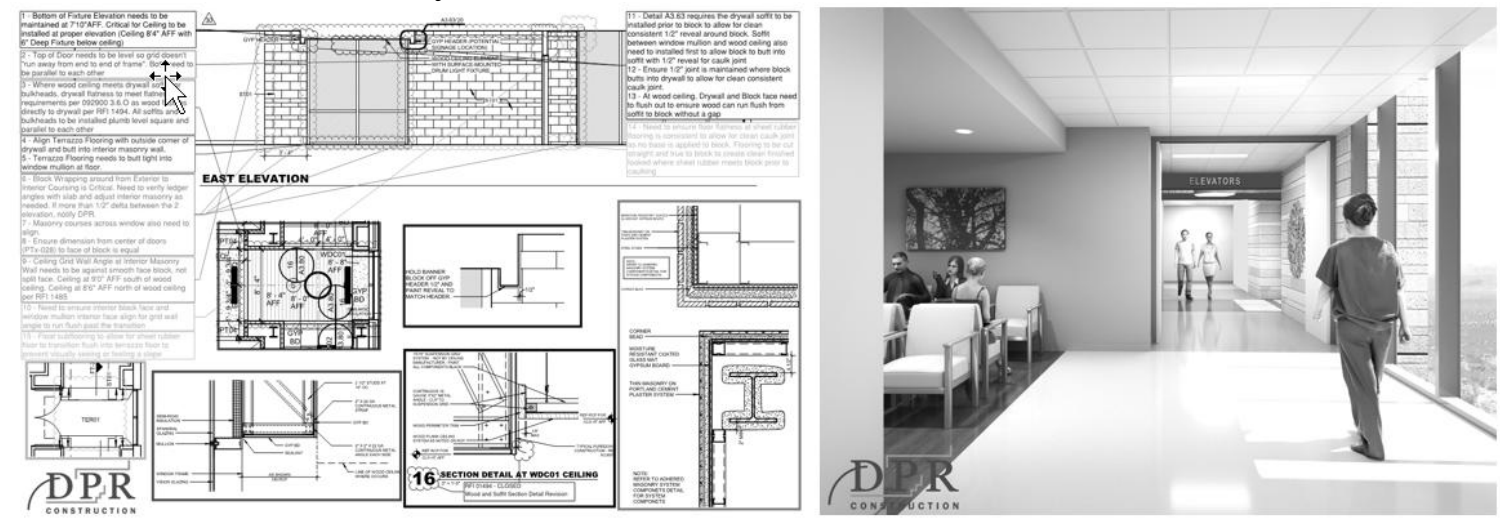

Figure 3: Medical Center Elevator Lobby Distinguishing Features of Work.

The process of identifying and aligning around DFOW organically focused all stakeholders on what was important and improved communication. DFOW and measurable acceptance visuals were posted on site. Before starting a DFOW area, kick- 
off meetings were held at the DFOW location to review the quality expectations. Foremen were asked to sign a copy of the DFOW and acceptance criteria.

On-site DFOW visuals also increased communication between trade partners. Trade partners were often seen gathering around DFOW visuals and coordinating amongst each other. It increased production, reduced rework, and increased trust between team members.

When the team completed drywall on the first floor of the patient tower, it resulted in no issues or punch list. The architect was so impressed with the quality process and results, he saw no need to walk the drywall scope again until the floor layout changed. The image on the left in Figure 3 shows how the GC team captured and communicated DFOW and acceptance criteria to the craft for the elevator lobby space. The rendering on the right in Figure 3 depicts the elevator lobby space. Both images were posted on site for the craft.

\section{PHARMACEUTICAL LAB}

Our final example shows how the Quality Workflow can be applied to any process or deliverable to produce predictable results and no surprises. That's because it becomes a way of thinking, a mindset. This project is a pharmaceutical lab project with a fast-paced scheduled. To meet our customer's timeline, the team had to present a fully coordinated building model around the same time as the design was scheduled to be complete. This necessitated an early involvement design-assist approach for the electrical, plumbing, and mechanical scopes of work.

Applying the Quality Work flow was crucial to the success of the project. The team developed DFOW for the design, specifically the information our trade partners would need from the design team, so they would only need to draw it once in the BIM. They also identified when information would be needed so as to complete modelling and start fabrication and installation; the Point of Release. The crucial element was the GC sitting with their trade partners' project management and field to develop a specific and measurable list of criteria for each design DFOW, along with dates the criteria was needed.

The results were intriguing. Although the modelling team did not receive all information they needed before every Point of Release, the project team was able to identify and implement mitigation plans beforehand to maintain the construction schedule. The design team was aware of why each identified piece of information was critical to the BIM process and understood the consequences of not providing the criteria before the Point of Release. Creating this understanding and communication developed trust and allowed the trade partners to adapt more quickly, and it added value to the coordination process. For example, the GC and trade partner knew early that a certain air valve would not be defined when requested. Instead of drawing air valves and redrawing them when they were specified, the team used a reasonable place holder approach that allowed work to proceed. Without this quality-based mindset, the coordination process would have halted or resulted in a significant amount of re-coordination. 


\section{CONCLUSION}

The paradigm shift for the construction industry will come from recognizing that quality is not just about a series of checks and checklists at key milestones. Quality Workflow goes beyond checklists in a powerful way. It forces people to talk, discuss, and eventually agree on what they will give and get, aligning expectations. Through those conversations, they move from implicit to explicit. Because they understand what they need to deliver, people can make better estimates of the time it will take for them to deliver. Planning, the first step in the Plan-Do-Check-Act (PDCA) cycle, improves. Wherever Quality Workflow has been implemented, results have been more predictable for the trades performing the work and for the customer.

Once people learn this approach, they quickly see that they can use it for all their tasks because it forces them to think about and agree on what is important about the work, and, most importantly, what they will provide to the customer. When this occurs, we say they have shifted to a "Quality Mindset," and have learned to focus first on meeting the customer's acceptance criteria. Implicit knowledge becomes explicit, and guides people's actions. The knowing-doing gap is closed and most of the challenges identified in the ACCI research are effectively addressed.

For many professionals, this approach can be challenging because it requires them to be vulnerable and perhaps admit that what they were planning to deliver might not have been clear to the suppliers and may not have aligned with customer expectations. In the traditional approach, where conversations about quality take place when looking at the installed work, there is room for plausible deniability. When the work is installed to undefined expectations, the owner or architect can take the position that "the contractor is experienced and should have known this isn't what we wanted" and the contractor can fall back on a standard defence: "How could we have known the implicit expectations? They weren't captured in the plans and specifications." These are "get out of jail free" cards that reflect a two-sided failure. Quality Workflow conversations are about making each stakeholder's assumptions explicit - and measurable - and creating a no excuse culture. It's about communicating in a way that builds trust and accountability and produces meaningful work.

Changing this GC's culture started with a simple vision and an elegant solution: a behavioral shift. The more they described why they were changing their approach — and how simply changing their focus and behaviors would have a tremendous impact on their ability to deliver work - the more their leaders also saw the benefit in challenging the status quo and moving to a predictable way to deliver a quality product. No worker wants to produce a product that is unacceptable.

\section{REFERENCES}

Burati, J., Farrington, J. \& Ledbetter, W. (1992). "Causes of quality deviations in design and construction." Journal of Construction Engineering and Management, Vol. $118(1)$.

Christian D., "Where Ambitious Owners with Ambitious Projects Need Ambitious GC's to Focus," unpublished presentation at the DPR Quarterly meeting, April 25, 2012. 
Dougherty, J. M., Hughes, N., and Zack, Jr., J. G. (2012). "The impact of rework on construction \& some practical remedies." Navigant Construction Forum ${ }^{\mathrm{TM}}$, Navigant, 21.

Hauser, J. R., and Clausing, D. (1988). "The House of Quality.” Harvard Business Review (May).

Ledbetter, W.B. (1994)."Quality performance on successful projects."Journal of Construction Engineering and Management, Vol. 120 (1).

Love P. (2002). "Auditing the indirect consequences of rework in construction: A case basedapproach."Managerial Auditing Journal, 17 (3), 138-146.

Marosszeky, M., Thomas, R., Karim K., and McGeorge D. (2002)."Quality management tools for lean production-moving from enforcement to empowerment."Proceedings IGLC-10, Bramado, Brazil.

Oakland, J. S., and Marosszeky, M. (2017). Total Construction Management: Lean Quality in Construction Project Delivery. Routledge, Abingdon, Oxon, Case Study 9 Safety, quality and BIM drive lean transformation at DPR, 549-551.

Ohno, T. (1988). Toyota production system: beyond large-scale production. Productivity Press Cambridge MA, 143p.

Pfeffer, J., and Sutton, R. I. (1999). The Knowing-Doing Gap: How Smart Companies Turn Knowledge into Action. Harvard Business Review Press.

Trethewy, R.W., Gardner, D., Cross, J., Marosszeky, M. (2000). "Behavioral Safety and Incentive Schemes." Proceedings of the Futurusafe 2000 Conference, Sydney, Australia.

"USC Michelson Center for Convergent Bioscience." (2017). DPR Construction, $<$ https://www.dpr.com/projects/michelson-center-for-convergent-bioscience>. 